\title{
Does analysis of bronchoalveolar lavage fluid provide a tool to monitor disease progression or to predict survival in patients with HIV-1 infection?
}

Human immunodeficiency virus type I (HIV-1), the aetiological agent of the acquired immunodeficiency syndrome (AIDS), causes slow but progressive destruction of the immune system. In addition to the loss of immune function, HIV-infected patients manifest various infectious and noninfectious pulmonary complications. In particular, despite improvements in therapy, Pneumocystis carinii pneumonia (PCP) still remains the most common cause of morbidity and mortality in patients with advanced HIV infection. ${ }^{1}$

Since the early years of the AIDS epidemic, analysis of bronchoalveolar lavage (BAL) fluid associated with transbronchial lung biopsy has been considered the procedure of choice for the evaluation of the full spectrum of pulmonary diseases throughout the course of HIV-1 infection. ${ }^{2}$ Apart from its diagnostic role, the analysis of cells in BAL fluid has greatly increased our knowledge of the inflammatory events taking place in the pulmonary microenvironment, as well as the effects of retrovirus on the host lung defence mechanisms. ${ }^{3}$ In particular, taking advantage of the availability of monoclonal antibodies, cell culture facilities, and the cloning of a number of immunologically relevant genes (including those encoding for cytokines and cytokine receptors), researchers have recently elucidated mechanisms underlying the accumulation of inflammatory cells in the lung. Furthermore, the introduction of the polymerase chain reaction (PCR), which permits specific amplification of discrete DNA sequences, has increased detection of proviral sequences in BAL fluid samples, thus answering some basic questions related to the cellular pattern of infectivity and the mechanisms behind the spread of HIV-1 into the lung.

Most studies of BAL fluid have contributed to the comprehension of events leading to the impairment of local immunocompetence. Recently, however, the attention of some groups has turned to defining the potential usefulness of BAL in providing prognostic information in patients with AIDS. In a time in which BAL has come of age, attempts are being made to correlate the cells (or cell products) in BAL fluid with clinical aspects of AIDSassociated interstitial lung disease. In an attempt to answer the provocative question raised in the title we will critically analyse studies claiming that BAL yields additional information predictive of diminished prognosis or survival for the patient infected with HIV-1. With the ultimate goal of confirming that the evolution of studies in this field continues on an appropriate track, we will also provide a framework for further investigation on the relation between immunological aspects of the interstitial inflammation and the respiratory disorders occurring as HIV-1 disease progresses. Finally, we will briefly summarise the first sets of observations covering the contribution of analysis of BAL fluid in monitoring treatment of AIDS-related pulmonary complications.

\section{Relation between disease progression and HIV-1 infection of lung cells}

Several techniques have been used to search for HIV-1 in cell populations isolated from the BAL fluid of patients with AIDS, including DNA-PCR, in situ hybridisation, p24 HIV protein detection in BAL cells and fluids. ${ }^{3}$ By these methods HIV-1 sequences and proteins have been detected in cells retrieved from BAL fluid of HIV-infected patients and the integration of the HIV-1 genome has been shown in alveolar macrophages, pulmonary $T$ cells, and fibroblasts. The mechanisms leading to the retroviral infection of the respiratory tract have also been elucidated to some extent. Peripheral blood CD4 + infected cells, predominantly monocyte macrophages that harbour the provirus in a latent form, may enter the lung where they differentiate into resident cells, thus allowing the persistence of the retroviral infection. In view of the recent suggestion that secondary lymphoid follicles of the lung serve as reservoirs for HIV-1, ${ }^{4}$ the establishment of HIV-1 infection of the lung may also be regarded as the consequence of a migratory process involving infected $\mathrm{T}$ cells originating from pulmonary lymphoid tissues.

Despite pulmonary complications which are characteristic of the advanced phases of HIV-1 infection, the lungs can be infected even in the asymptomatic period. This concept is substantiated by the observation that HIV1 sequences can be demonstrated in pulmonary cell populations recovered from the respiratory tract of HIV-1 seropositive subjects at all stages of infection, albeit at different levels ${ }^{3}$ and influenced by several factors. ${ }^{56}$ HIV-1 can be more readily detected in the BAL fluid of individuals with PCP than in patients with non-PCP lung infections, while airway superinfection with cytomegalovirus does not affect the isolation rate of HIV-1. Retroviral sequences are found more frequently in the lungs of individuals receiving no antiviral chemotherapy than in those receiving treatment with zidovudine (AZT), and more frequently in smokers than in non-smokers. ${ }^{6}$ Since the retrovirus can easily be demonstrated in pulmonary cell populations of patients undergoing a respiratory episode, ${ }^{5}$ virology studies have claimed to provide relevant information in terms of prognosis and survival. It has been also observed that HIV-1 detection in BAL fluid samples is significantly associated with progression to death ${ }^{5}$ but not to reduction of pulmonary function tests. ${ }^{7}$

On the basis of these data it is tempting to speculate that the switch from viral latency to productive infection and the emergence of virulent HIV-1 variants might have profound effects on changes in pulmonary function and survival of patients with AIDS. Detailed analyses are needed to characterise HIV-1 strains that complete reverse transcription in the lung in vivo. These data are central to verifying whether the emergence of retroviral variants represents an important factor affecting the pulmonary manifestation of HIV-1 infection. Furthermore, in view of the genetic instability of HIV $-1,{ }^{8}$ efforts should be made to determine whether the well known person-to-person genomic variation of HIV-1 influences the development of clinical manifestations and the disease progression of respiratory illness during HIV-1 infection. Furthermore, since cytokines secreted during the pulmonary immune response against HIV-1 may initiate the spread of HIV-1 infection (see below), the understanding of the contribution of BAL cellular factors in increasing HIV-1 replication will be useful for the development of effective programmes that address the therapeutic needs of HIV-1 infected subjects. 


\section{Influence of inflammatory response of host on disease progression and survival}

The issue of the immunological events occurring in the lung of HIV-1 infected patients is beyond the scope of this editorial. Current concepts on how the pulmonary immune system attempts to control HIV-1 spread, and on the role of the retrovirus in determining the functional impairment of the pulmonary host defence mechanisms, have been covered in recent reviews. ${ }^{39}$ Here we will only briefly mention the mechanisms accounting for the AIDSassociated alveolitis in defining the link between the local immunoregulatory networks and the development of respiratory failure.

IMMUNOLOGICAL ABNORMALITIES IN THE LUNG OF HIV-1 INFECTED PATIENTS

The presence of HIV-1 infected cells in the pulmonary microenvironment elicits a significant local immune response. In fact, about $25 \%$ of subjects with early infection and $50 \%$ of patients with advanced disease show a high intensity alveolitis that is initiated and sustained by HIV1 specific cytotoxic T lymphocytes, ${ }^{10}$ natural killer cells, ${ }^{11}$ and alveolar macrophages and mediated by a number of cytokines $^{3}$; the number of alveolar neutrophils is normal in asymptomatic patients but can increase in a subset of patients with opportunistic infections.

The inflammatory process of the airway is also documented by the presence of non-specific indicators of inflammation in cell-free BAL fluid. An increased recovery of high molecular weight proteins has been documented in the BAL fluid of patients with AIDS, including $\alpha_{2}$ globulin. ${ }^{12}$ The surfactant composition is also altered and high concentrations of immunoglobulins, including antibodies specific to HIV-1, and immune complexes are commonly present in lung epithelial lining fluid of these patients. ${ }^{31314}$ It is also believed that the presence in BAL fluid of triggering molecules and activation products of the coagulation and fibrinolytic system might reflect a milieu that favours the accumulation of fibrin in inflammatory lung tissues. ${ }^{15}$ As a further confirmation of this concept, serum levels of the amino-terminal propeptide of type III procollagen, a marker of tissue damage that mirrors inflammatory activity and fibrogenesis in involved organs, are increased in patients with advanced PCP. ${ }^{16}$

A question that has recently generated much debate concerns the regulatory networks between HIV-1, viruses superinfecting pulmonary interstitium, and the in situ release of cytokines. Several agents have been shown to upregulate the HIV-1 expression in cells chronically or latently infected by the retrovirus. ${ }^{17}$ For instance, opportunistic viral pathogens that can be isolated from the lung of patients with AIDS may interact with HIV-1 encoding trans-activator proteins. Furthermore, it has been shown that pulmonary cells from HIV-1 infected individuals spontaneously release biological mediators of the immune response, including interleukin 2 (IL-2), IL-6, IL-8, tumour necrosis factor $\alpha$ (TNF $\alpha$ ), and granulocytemacrophage colony stimulating factor (GM-CSF). ${ }^{3}$ These cytokines, besides eliciting activation of local immunocompetence, may paradoxically increase HIV-1 expression, ${ }^{17}$ suggesting that the dissemination of HIV-1 could be initiated by viral and cellular factors that are involved in the pathogenesis of intrapulmonary inflammatory lesions. Once again, these findings suggest the need for large scale studies that will sequentially measure cytokine levels in the BAL fluid of patients at various stages of the disease and/or with different types of opportunistic infections.
LINK BETWEEN LOCAL INFLAMMATORY PROCESSES AND THE DEVELOPMENT OF RESPIRATORY FAILURE

Abnormalities of pulmonary function are present not only in HIV-1 seropositive patients with diffuse disease of the lung parenchyma but also in symptomless individuals without abnormalities on chest radiographs. ${ }^{18}$ In particular, frequent abnormalities throughout the course of HIV infection are a low carbon monoxide diffusing capacity caused by an impairment of the exchanging ability of the alveolar capillary membrane, and an abnormal arterial oxygen tension that is present in virtually all patients with PCP after exercise. Patients infected with HIV-1 but without identifiable lung infection or neoplasm also have a reduced mean carbon monoxide transfer factor (TLCO) and accelerated clearance of diethylene triamine pentaacetate (DTPA). Interestingly, abnormal TLCo in the absence of lung disease represents a marker of HIV-1 induced immunosuppression and is therefore a predictor for a more rapid progression to AIDS. ${ }^{19}$

The finding that HIV-1 seropositive individuals with no evidence of lung disease may have a high intensity alveolitis associated with a reduction in TLCO supports the suggestion that local inflammation plays a part in the progressive decline of pulmonary function. This line of reasoning implies that the respiratory epithelium may represent a potential target for effector $\mathrm{T}$ cells which, during immunemediated response to HIV-1, are recruited from blood to the interstitium and then to the epithelial surface. Furthermore, a strong correlation exists between the extent of CD8 + alveolitis and the value of epithelial permeability, as determined by the pulmonary clearance of inhaled ${ }^{99 \mathrm{~m}} \mathrm{Tc}$-labelled DPTA-Cl. ${ }^{20}$ Abnormalities of gas transfer coefficient (KCO) and $\mathrm{PaO}_{2}$ are also independently associated with the increase in the absolute number of alveolar CD8 cells. ${ }^{20}$

CD8 + cytotoxic T lymphocytes play an important, but not exclusive, part in the pathogenesis of respiratory failure. In fact, patients with severe PCP and a high neutrophil count in the BAL fluid show a high protein concentration and the presence of $\alpha_{2}$-globulin in the BAL fluid; the increase in levels of neutrophils and high molecular weight protein correlates with gas exchange abnormalities and improves as PCP resolves. ${ }^{12}$ This suggests that neutrophil products (including superoxide anion and proteolytic enzymes) released in response to $\mathrm{HIV-1}$ infection and/or the presence of opportunist infections could also be directly toxic to epithelial cells.

The pathogenetic role of alveolar macrophages in the events leading to lung injury is, for the time being, hypothetical. Because TNF $\alpha$ is able to alter the lung endothelium causing oedema and interstitial damage, it is likely that this proinflammatory cytokine directly contributes to the decline in pulmonary function. Furthermore, since TNF $\propto$ production by alveolar macrophages of seropositive patients is significantly related to the expression of HIV-1 by these cells, ${ }^{21}$ it is possible that the vicious cycle existing between the retrovirus and TNF $\alpha$ generates a pool of alveolar macrophages refractory to any further stimulation; it is conceivable that this condition may favour the development of additional pulmonary infections, exposing the host to the risk of respiratory failure. Considering the potential clinical problems associated with a local exaggerated release of TNF $\alpha$, one must question the relative importance of the determination of TNF $x$ levels in BAL fluid in assessing the risk of the progressive loss of lung function. This will be a primary issue for the future that must be resolved prospectively by testing levels of TNF $\alpha$ in BAL fluid in a cohort of patients with HIV-1 infection and variable clinical and immunological status.

Alveolar macrophages may also indirectly participate in 
lung injury by synthesising neutrophil chemotaxins. In this regard, attention has recently been paid to the fact that, as a result of their persistent activation, alveolar macrophages release high levels of IL-8 and GM-CSF locally. ${ }^{223}$ Even more interesting is the finding that levels of IL-8 and GMCSF in BAL fluid strongly correlate with the neutrophil count. ${ }^{223}$ Furthermore, determination of levels of IL-8 in BAL fluid seems to be a sensitive method for the clinical follow up of respiratory illness in patients with HIV-1 infection because a significant correlation between changes in IL-8 levels and differences in alveolar arterial oxygen pressure can be observed. ${ }^{23}$ Whether longitudinal decline in gas exchange is related to the levels of GM-CSF in BAL fluid is still unknown and needs appropriate investigation. Furthermore, studies must be designed to assess critically the prognostic use of the levels of other cytokines in the BAL fluid that are involved in the attraction of inflammatory cells (including G-CSF and monocyte chemotactic protein 1) or that locally promote fibrin deposition through the induction of procoagulant activity (including IL-1, TGF- $\beta$, or IFN $\gamma$ ).

\section{ROLE OF TREATMENT IN THE IMPROVEMENT OF} RESPIRATORY FUNCTION

It is now well established that early adjunctive treatment with steroids reduces the risks of respiratory failure and death in patients with AIDS and moderate to severe PCP. ${ }^{24}$ Huang and $E_{d e n}{ }^{25}$ have recently shown that steroids lower the release of IL-1 $\beta$ and TNF $\alpha$ by cultured alveolar macrophages from patients with AIDS in a dose-dependent fashion. On this basis they proposed that the drugs improve the outcome of AIDS-associated pulmonary complications by inhibiting the in situ release of proinflammatory cytokines. By controlling the damaging activity of the local immune system, steroids could restore alveolar epithelial and vascular endothelial integrity, shifting the balance towards an improvement of the clinical condition..$^{25}$ The capability of prednisone for modulating the transcription rate of lymphokines (including IL-2) raises the alternative hypothesis that steroids favour the recovery of pulmonary function by blocking the accumulation and the functional activities of $\mathrm{CD} 8+$ cytotoxic $\mathrm{T}$ lymphocytes. Additional studies are needed to determine whether these molecules lead to a decreased production of those lymphokines that are required for the growth and function of pulmonary cytotoxic $\mathrm{T}$ lymphocytes in the lung.

Another issue that needs to be resolved in the near future is related to the importance of antiretroviral treatment in the modulation of local immunocompetence. It has been observed that AZT reverses impaired expression of phenotypic markers on alveolar macrophages of patients with AIDS. ${ }^{26}$ In particular, following antiretroviral treatment the alveolar macrophage expression of HLA-DR molecules and RFD1 markers (two determinants that are closely associated with the functional capabilites of alveolar macrophages) returns to levels of expression seen in normal lavages. These data emphasise that alveolar macrophage function must be monitored in patients who receive AZT. In particular, it would be important to determine whether antiretroviral therapy modifies the pattern of cytokine release in infected cells, thus reducing the risk of a TNF $\alpha$ mediated respiratory failure.

SIGNIFICANCE OF BAL CELL POPULATIONS IN PREDICTING SURVIVAL

The relatively high incidence of respiratory failure in patients with intrapulmonary inflammatory lesions raises the central issue of whether the study of cell and cell products

in BAL fluid provides additional information relevant to survival among HIV-1 infected patients. The prognostic use of morphological and immunological evaluation of BAL cell populations in determining the mortality risk in HIV-1 infection has been considered by several groups. ${ }^{27-29}$ The risk of death is greatly increased in HIV-1 seropositive individuals with opportunistic infections and BAL neutrophilia, whereas in patients without histological, culture, or cytological confirmation of pulmonary opportunistic infections, the most important variable with a predictive value appears to be the number of pulmonary $\mathrm{CD} 8+$ lymphocytes.

Although the studies quoted above outline the predictive value of BAL cell analysis, they were based on a retrospective evaluation of data obtained from selected groups of patients belonging to the IV CDC group who developed a fatal opportunistic infection after a variable period following analysis of BAL fluid. Also, the small number of patients made it impossible to obtain highly statistically significant values. Even more importantly, information on changes of individual parameters in BAL fluid with tim.. are still lacking. Longitudinal studies of BAL fluid findir:in a large number of HIV-1 infected patients followed from an asymptomatic stage until the diagnosis of AIDS are necessary to define clearly the natural course of the respiratory illness in HIV-1 infection.

A cooperative study has recently been designed to examine the prognostic impact of BAL analysis in a large cohort of patients from centres in nine European countries. The purpose of this European project is to collect data on the BAL fluid from a large group of HIV-1 infected patients from different countries and infected by different opportunistic agents. The study is concerned specifically with evaluating the importance of BAL parameters in determining the mortality and morbidity risk. However, BAL fluid data will be correlated with a number of demographic, clinical, and immunological variables through a multivariate analysis. We hope that this approach will provide a risk stratification among HIV-infected patients, raising the possibility of using BAL cell findings and other markers, such as significant demographic and clinical features, viral burden, or cytokine levels in the BAL fluid, to develop a scoring system. This will facilitate early recognition of cases who are at the greatest risk of death, and thus most likely to benefit from early intervention with therapeutic agents including pentamidine and/or other prophylactic agents which may prevent pulmonary complications. Given the high morbidity and mortality associated with the development of pulmonary complications in an asymptomatic HIV-1 seropositive patient, it will also be important to assess the validity and the possibility of extending the use of these prognostic determinants to individuals with early HIV-1 infection. Such approaches should provide predictive information in a timely manner for those patients infected with HIV-1 who are at risk of developing disabling illnesses, without subjecting those with a low or no risk for respiratory complications to unnecessary discomfort.

\footnotetext{
The authors wish to thank their colleagues from the Departments of Infectious Diseases and Pulmonary Medicine of the Padua Hospital, in particular Drs P Cadrobbi and A Cipriani, who contributed to the original studies discussed in this paper by allowing the study of their patients and performing the bronchoscopy. We also wish to thank Mr Martin Donach for his help in the preparation of the manuscript. Supported by Grants from the Ministero della Sanità, Istituto Superiore della Sanità, Progetto AIDS, 1994 (Rome).

Reprint requests to: Dr G Semenzato. 
1 Murray JF, Mills J. Pulmonary infectious complications of human immunodeficiency virus infection. Part I and Part II. Am Rev Respir Dis $1990 ; 141: 1356-72,1582-98$

2 Broaddus VC, Dake MD, Stulbarg MS. Bronchoalveolar lavage and transbronchial biopsy for the diagnosis of pulmonary infections in the acquired immunodeficiency syndrome. Ann Intern Med 1985;102:742-52.

3 Agostini C, Trentin L, Zambello R, Semenzato G. State of the art. HIV-1 and lung. Infectivity, pathogenic mechanisms and cellular immune response taking place in the lower respiratory tract. Am Rev Respir Dis 1993; 147:1038-41.

4 Pantaleo G, Graziosi C, Fauci AS. The immunopathogenesis of human immunodeficiency virus infection. $N$ Engl F Med 1993;328:327-35.

5 Clarke JR, Taylor IK, Fleming J, Nukuna A, Williamson JD, Mitchell DM. The epidemiology of HIV-1 infection of the lung in AIDS patients. AIDS 1993;7:555-60.

6 Clarke JR, Williamson JD, Mitchell DM. Comparative study of the isolation of human immunodeficiency virus from the lung and peripheral blood of AIDS patients. F Med Virol 1993;39:196-9.

7 Clarke JR, Fleming J, Donegan K, Moss FM, Nieman R, Williamson JD, et al. Effect of HIV-1 and cytomegalovirus in bronchoalveolar lavage cells on the transfer factor for lung carbon monoxide in AIDS patients. AIDS 1991;5:1333-8.

8 Schuitemaker H, Koot M, Kootstra NA, Dercksen MW, De Goede REY, van Steenwijk RP, et al. Biological phenotype of human immunodeficiency virus type 1 clones at different stages of infection: progression of disease virus type 1 clones at different stages of infection: progression of disease is associated with a shift from mon
populations. $f$ Virol 1992;66:1354-60.

9 Mayaud CM, Cadranel J. HIV in the lung: guilty or not guilty. Thorax 1993;48:1191-5.

10 Autran B, Mayaud C, Raphael M, Plata F, Denis M, Bourguin A, et al. Evidence for a cytotoxic $T$ lymphocyte alveolitis in human immunodeficiency virus-infected patients. AIDS 1988;2:179-83.

11 Agostini C, Zambello R, Trentin L, Feruglio C, Masciarelli M, Siviero F, et al. Cytotoxic events taking place in the lung of patients with HIV-1 infection. Evidence for an intrinsic defect of the MHC-unrestricted killing infection. Evidence for an intrinsic defect of the MHC-unrestricted killing partially rest
142:516-22.

12 Sadaghdar H, Huang ZB, Eden E. Correlation of bronchoalveolar lavage findings to severity of Pneumocystis carinii pneumonia in AIDS. Evidence for the development of high-permeability pulmonary edema. Chest 1992 102:63-9.

13 Young KR, Rankin JA, Naegel GP, Paul ES, Reynolds HY. Bronchoalveolar lavage cells and proteins in patients with the acquired immunodeficiency syndrome: an immunological analysis. Ann Intern Med 1985;103:522-33.

14 Nowakowski M, Clarke LM, Amaro R, Pellegrino MG, Sierra MF, Steiner $P$. Characterization of cells, immunoglobulins, and immune complexes
present in the bronchoalveolar lavage of pediatric AIDS patients. Reg present in the broncho

15 Nakstad B, Lyberg T, Skjonsberg OH, Boye NP. Local activation of the coagulation and fibrinolysis systems in lung diseases. Thromb Res 1990; 57:827-38.

16 Bentsen KD, Nielsen TL, Eaftinck Scattenkerk JKM, Jensen BN, Lundgren JD. Serum type III procollagen peptide in patients with Pneumocystis carinii infection. Am Rev Respir Dis 1993;148:1558-62.

17 Cullen BR. Regulation of HIV-1 gene expression. FASEB f 1991;5:2361-8.

18 Mitchell DM, Fleming J, Pinching AJ, Harris JR, Moss FM, Veale D, et al. Pulmonary function in human immunodeficiency virus infection. $A m R e v$ Respir Dis 1992;146:745-51.

19 Nieman RB, Fleming J, Coker RJ, Harris JR, Mitchell DM. Reduced carbon monoxide transfer factor ( $T_{L C O}$ ) in human immunodeficiency virus type I (HIV-I) infection as a predictor for faster progression to AIDS. Thorax 1993;48:481-5.

20 Meignan M, Guilon JM, Denis M, Joly P, Rosso J, Carette MF, et al Increased lung epithelia permeability in HIV-infected patients with isolated cytotoxic T lymphocytic alveolitis. Am Rev Respir Dis 1990;141:1241-8.

21 Israel-Biet D, Cadranel J, Beldjord K, Andrieu M, Jeffrey A, Even P. Tumor necrosis factor production in HIV-seropositive subjects. Relationship with necrosis factor production in HIV-seropositive subjects. Relationship with f Immunol 1991;147:490-4.

22 Agostini C, Trentin L, Zambello R, Bulian P, Caenazzo C, Cipriani A, et al. Release of GM-CSF by alveolar macrophages in the lung of HIV-1 infected patients. A mechanism accounting for macrophage and neutrophil accumulation. F Immunol 1992;149:3379-85.

23 Lipschik GY, Doerfler ME, Kovacs JA, Travis WD, Andrawis VA, Lawrence MG, et al. Leukotriene B4 and interleukin-8 in human immunodeficiency virus-related pulmonary disease. Chest 1993;104:763-9.

24 Bozzette SA, Sattler FR, Chiu J, Wu AW, Gluckstein D, Kemper C, et al. A controlled trial of early adjunctive treatment with corticosteroids for Pneumocystis carinii pneumonia in the acquired immunodeficiency syndrome. California Collaborative Treatment Group. $N$ Engl f Med 1990; 323:1451-7.

25 Huang ZB, Eden E. Effect of corticosteroids on IL-1 beta and TNF alpha release by alveolar macrophages from patients with AIDS and Pneumocystis release by alveolar macrophages from patie

26 Bray DH, Squire SB, Kawana A, Johnson MA, Poulter LW. Antiretroviral treatment reverses HIV-induced reduction in the expression of surface antigens on alveolar macrophages in AIDS patients. Clin Exp Immunol 1993;91:13-7.

27 Agostini C, Zambello R, Trentin L, Poletti V, Spiga L, Gritti F, et al. Prognostic significance of the evaluation of bronchoalveolar lavage populations in patients with HIV-1 infection and pulmonary involvement. Chest 1991;100:1601-6.

28 Mason GR, Hashimoto CH, Dickman PS, Foutty LF, Cobb CJ. Prognostic implications of bronchoalveolar lavage neutrophilia in patients with Pneumocystis carinii pneumonia and AIDS. Am Rev Respir Dis 1989; 139:1336-42

29 Jensen BN, Lisse IM, Gerstoft J, Borgeskov S, Skinhoj P. Cellular profiles in bronchoalveolar lavage fluid of HIV-infected patients with pulmonary symptoms: relation to diagnosis and prognosis. AIDS 1991;5:527-33. 